\title{
Propiedades psicométricas de un cuestionario para la evaluación de la calidad de vida urbana y el miedo al delito
}

\author{
César San Juan iD, Anabel Vergara iD e Isabel Germán iD \\ Instituto Vasco de Criminología
}

\section{RESUMEN}

En este trabajo se muestran los resultados parciales de un estudio de sociológico desarrollado en el País Vasco. Se presentan las propiedades psicométricas de diversas escalas útiles para evaluar la Calidad Urbana y el Miedo al Delito. Por otra parte, se contrasta una propuesta de modelo explicativo de Miedo al Delito en el que son cuestionados los modelos de Percepción de riesgo, Victimación y Vulnerabilidad en detrimento de una perspectiva más racionalista.

Palabras clave: Percepción de inseguridad, Miedo al delito, Evaluación medio urbano.

\section{ABSTRACT}

In this work we show the results of a sociological study developed in the Basque Country. We show the psychometric properties of several useful scales for assessing the Urban Quality and the Fear of Crime. In the other hand, a proposal of a Fear of Crime predictive model is contrasted. This model questions the Risk Perception, Victimization and Vulnerability Models against a rationalist perspective. A model of the Urban Insecurity Perception is contrasted.

Key words: Insecurity perception; Fear of crime, Urban environment evaluation.

\section{Introducción}

Cuando se elaboran estudios sobre percepción de sentimiento de inseguridad y miedo al delito, pueden presentarse algunos problemas metodológicos con importantes repercusiones en el planteamiento y análisis de los datos (Fattah, 1993). Más concretamente a la hora de examinar la relación entre el miedo al delito y variables psicológicas, ambientales, sociales, políticas y económicas. Constituye un apasionado tema de debate en la literatura decidir cuáles son las variables con una mayor importancia en este tipo de investigaciones (Kury y Ferdinand, 1999). Las relaciones entre edad, sexo, grupo étnico, ingresos, estado civil y miedo al delito son controvertidas, ya que se han alcanzado resultados muy diferentes, incluso contradictorios (Hale, 1996). Dicha circunstancia dificulta enormemente todos los intentos de realizar una sistematización teórica que pueda ser útil a la hora de tomar decisiones con respecto a las políticas de seguridad y prevención del delito. A pesar de que junto al sexo, la edad es la variable que ha sido estudiada con mayor frecuencia en relación con el miedo al delito en la literatura criminológica de los años 80 (Eve, 1985; Lagrange \& Ferraro, 1989; Stafford \& Galle, 1984; Warr, 1984), nosotros pensamos que lo más importante no consiste en buscar diferencias categóricas entre los diferentes

\footnotetext{
*Esta investigación fue financiada por la Consejería de Interior del Gobierno Vasco.

Revista Española de Investigación Criminólogica

REIC AC-01-05 http://www.criminologia.net

https://doi.org/10.46381/reic.v3i0.22
}

ISSN 1696-9219 
colectivos sociales sino, sobre todo, intentar explicar qué es exactamente el miedo al delito y por qué se siente la gente insegura en los hábitat urbanos (San Juan, 2001).

En este sentido, y desde hace ya varios años, una de las cuestiones más debatidas ha sido la denominada "paradoja del miedo al delito" (Lindquist \& Duke, 1982), según la cual aquellos grupos entre los que el sentimiento de inseguridad y miedo al delito es mayor, concretamente mujeres y ancianos, son los que sufren un número de victimizaciones más bajo. Mientras que, por el contrario, hombres y jóvenes adultos, a pesar de ser los que mayor número de victimizaciones sufren, son los que menos miedo padecen.

La ambigüedad en los resultados convierte esta línea de investigación en un "caso abierto" precisamente en un momento en el que la medición de la percepción de inseguridad y el miedo al delito se han convertido en variables imprescindibles en los estudios de calidad de vida. Parece conveniente, por tanto, además del necesario esfuerzo que hemos citado de aclarar los términos, disponer de una herramienta útil tanto para la investigación básica como para los observatorios e instituciones encargados de diseñar las políticas locales de seguridad (Díez Ramos, 1996).

\section{Procedimiento y Muestra}

El cuestionario fue confeccionado ad hoc para este estudio, aunque algunas de sus secciones están basadas en otros trabajos como se irá especificando en el apartado de resultados. El trabajo de campo fue realizado durante los meses de noviembre y diciembre del año 2003 por una empresa especializada en estudios de opinión subcontratada por el Instituto Vasco de Criminología.

En cada una de las tres capitales de la Comunidad Autónoma Vasca, -Bilbao, Vitoria y San Sebastián,- se ha diseñado una muestra independiente siguiendo un procedimiento estratificado y polietápico por conglomerados en cuanto a la selección de las "secciones censales" donde realizar las encuestas. Y en cada una de dichas "secciones censales" se han seleccionado las personas a entrevistar (en su domicilio) siguiendo rutas aleatorias, a partir de una dirección de partida establecida de antemano, y cumpliendo cuotas de sexo, edad y actividad. El tiempo medio de respuesta de las entrevistas era 20 minutos.

La estratificación se ha realizado en función de una tipología de secciones, de cuatro o cinco categorías, elaborada al efecto y la pertenencia de la muestra a los estratos se ha realizado de forma proporcional a su población.

Se ha entrevistado a 504 personas de cada municipio en un conjunto de 9 barrios, tres de cada municipio, seleccionados por su tipología sociodemográfica, siendo el primer tipo correspondiente a lo que podríamos categorizar como nivel social alto, el segundo tipo, nivel social medio y el tercer tipo, nivel social bajo lo que hace un total de 1512 encuestas para una población global de 751.906 personas.

El error muestral para los resultados del conjunto de la muestra (1512 encuestas) será del $\pm 2,8 \%$ con un nivel de confianza del $95,5 \%$ para las proporciones más desfavorables del $50 \%$, del $\pm 4,5 \%$ para los resultados de cada una de las tres capitales partiendo de la hipótesis que los tres perfiles sociodemográficos representan la población de cada ciudad y, finalmente, del 7,5\% para cada uno de los 9 barrios tipo seleccionados.

El género está equilibrado al 50\% estableciéndose la mediana de edad en los 45 años. El 30,8 \% de las personas entrevistadas nació fuera de la Comunidad Autónoma

Revista Española de Investigación Criminólogica

REIC AC-01-05

http://www.criminologia.net

ISSN 1696-9219 
Vasca. Un $29 \%$ de personas solteras, un $59,3 \%$ de casadas, un 3,5\% de separadas o divorciadas y un $8,1 \%$ de personas viudas conforman el perfil del estado civil de la muestra. Por otra parte, y en lo que concierne al régimen de convivencia, un 19,6\% de personas convive con sus padres, un $22,2 \%$ vive en pareja sin hijos, un $39,3 \%$ vive con su pareja e hijos, un 2,4\% convive con amigos o amigas, un 9,9\% son personas que viven solas $\mathrm{y}$, por fin, un $6,5 \%$ de personas no se encuentran en ninguna de las situaciones descritas, de lo que puede deducirse que la mayor parte de este grupo son personas que no tienen pareja pero viven con uno o más hijos.

El nivel de instrucción es el siguiente: un 11,1\% de las personas entrevistadas no tiene estudios o tiene alguna formación inferior a los estudios primarios. El 26,6\% tiene estudios primarios, el $16,7 \%$ algún tipo de formación profesional, un porcentaje parecido, el 16,5\% dispone de estudios secundarios y, por fin, un $28,6 \%$ ha alcanzado una diplomatura o estudios superiores. En relación con la actividad principal, vemos que la mayor parte de las personas entrevistadas, un $40 \%$ trabaja por cuenta ajena, seguidos de un $19,8 \%$ de personas que se dedican a labores del hogar y un porcentaje parecido, el 19,2\% que son jubilados o pensionistas. El 5,4\% dicen estar sin empleo y un $9,1 \%$ fueron los estudiantes entrevistados.

\section{Resultados}

\subsection{Escala de Relación con el barrio: sentido de pertenencia y seguridad}

Como señala Naredo (2000), el sentimiento de inseguridad tiene una estrecha relación con la incomunicación y con el abandono de los espacios públicos. Este repliegue de los ciudadanos hacia lo privado hace que se limite el contacto con las personas del entorno y se pierda el control social sobre los espacios. En palabras de Lofland (1973), al referirse a la ciudad contemporánea, nos encontraríamos con "un mundo de extraños" donde nadie conoce a nadie. En este sentido, creemos que puede ser interesante la incorporación de aspectos relacionados con el sentido psicológico de barrio en un instrumento de análisis de la percepción de inseguridad y miedo al delito.

La fiabilidad de esta escala de Satisfacción con el Barrio es relativamente alta $(\alpha=0,77)$ y su validez para posteriores análisis la podemos contrastar con un nítido análisis factorial en el que encontramos cuatro factores (Tabla 1 en el apéndice). El primer factor está relacionado con la Satisfacción con Vivienda, Barrio y Vecinos (\% var.: 20\%),tres unidades fundamentales para el estudio de la Satisfacción Residencial (Amérigo, 1995). El segundo factor hace referencia a los Servicios del Barrio (\% var.:16\%), el tercer factor lo podemos identificar con la satisfacción con los Espacios Públicos. (\% var: 16\%). Y, el cuarto y último factor está relacionado con las Características Crimífugas de las calles (\% var: 14\%).

Como medida complementaria comprobamos que la variable "Sentido de pertenencia al barrio" presentaba una correlación de $0,31 \quad(\mathrm{p}<.000)$ con el factor de Satisfacción residencial y de 0,22 ( $<<.000)$ con el factor de Servicios del Barrio. En este sentido cabe destacar que la correlación de Sentido de Pertenencia con Satisfacción Residencial mejora la correlación de aquella con Tiempo de residencia en el Barrio $(0,30 ; \mathrm{p}<.000)$. 


\subsection{Tipología de lugares peligrosos}

La identificación de un lugar como potencialmente peligroso tiene que ver con aspectos que van más allá de la experiencia de victimación en ese espacio concreto. Con esta escala de Tipología de lugares peligroso (Fernández, 1996, 1997) en la que hemos encontrado un coeficiente de fiabilidad interesante $(\alpha=0,83)$, se pretende obtener un perfil de las características que convierten a un lugar como un espacio crimípeto en contraposición a lo que sería un espacio crimífugo. Estos conceptos han sido formulados en trabajos anteriores (San Juan, 2000) en los que se ha entendido por espacio crimípeto aquel escenario urbano que por sus especiales características físicas y arquitectónicas pudiera favorecer la comisión de ciertos delitos; en sentido contrario, un espacio denominado crimífugo inhibiría la comisión de delitos. El grado de visibilidad, presencia de control social, posibilidades de mantenerse oculto, o la facilidad para la huida, serían variables a tener en cuenta para establecer esta distinción.

Se realizó un análisis factorial de esta escala que ha tenido como resultado la aparición de tres factores de gran operatividad para potenciales análisis (Tabla 2 en el apéndice). Podríamos identificar el primer factor como Percepción de Peligro $(\alpha=$ 0,81 ), con una varianza explicada del 27\%, y el segundo factor como Características Espaciales Crimípetas $(\alpha=0,81)$, con una varianza explicada del $25 \%$. Este factor, relativo a las características espaciales crimípetas, tiene una correlación de 0,11 $(\mathrm{p}<.000)$ con la subescala de Satisfacción con los espacios públicos. Esto revela, precisamente, que cuanto mayor es dicha satisfacción, en mayor medida se reconoce la degradación del espacio como un síntoma de peligrosidad.

El último ítem, aparece individualmente, señalando la experiencia directa del entrevistado con el lugar en cuanto a la probabilidad de frecuentarlo. Ya que no aporta un porcentaje significativo de varianza explicada (10\%), podría ser conveniente eliminarlo de la subescala,- ya que el coeficiente alfa de fiabilidad aumentaría a 0,86,aunque no del cuestionario global porque informa de la relación que el entrevistado mantiene con el lugar considerado como potencialmente peligroso.

Mediante un análisis de regresión $\left(\mathrm{R}^{2}=44 \%\right)$ comprobamos que, dentro de esta escala, lo que mejor explica el ítem Al pasar o ir por ese lugar, a veces pienso que puedo ser víctima de un delito, es Normalmente suele haber gente con mala pinta $(\beta=0,15 ; \mathrm{p}=0,000) ;$ Es un lugar donde se suelen cometer delitos $(\beta=0,16 ; \mathrm{p}=0,000)$ y En caso de peligro es posible que nadie me prestase ayuda $(\beta=0,33 ; \mathrm{p}=0,000)$. Es destacable, por tanto, la importancia que tienen variables como la presencia de extraños, y las redes sociales, esto es, el sentido de territorialidad, en la identificación de un lugar como peligroso.

\subsection{Miedo a ser víctima de un delito}

A las personas entrevistadas se les solicita que valoren el grado de temor o miedo a que, personalmente, puedan ser víctimas de algún tipo de situación ansiógena o delictiva en una escala Likert de 0 a 10 . Sin duda lo más llamativo de esta respuesta es que las puntuaciones medias más elevadas aparecen en ser víctima de un acción terrorista (6,2), y la posibilidad de sufrir un accidente de tráfico $(6,5)$. Se trata de un dato ciertamente preocupante que la población del estudio tenga el mismo miedo o temor a sufrir una y otra situación. En este sentido, un análisis de varianza de medidas repetidas con el fin de comprobar las diferencias intrasujeto, revela un efecto Revista Española de Investigación Criminólogica 
estadísticamente significativo $(\mathrm{F}(6,8700)=145,16 ; \mathrm{p}=0,000)$. Las comparaciones múltiples entre pares de medias se realizaron mediante pruebas $t$ de Student para muestras relacionadas utilizando como valor crítico el de la distribución de módulo máximo estudentizado con $\mathrm{J}^{*}=21$ y g.l. $=1450$. Los resultados obtenidos indicaron que los sujetos manifiestan un mayor temor a las acciones terroristas, a un atraco o agresión en la calle, a ser robado en su domicilio y, como ya hemos apuntado, a la posibilidad de sufrir un accidente de trafico, que al resto de las situaciones que se describen en la escala. Por su parte, podemos confirmar que el aumento de la llegada de inmigrantes es la situación que menos temor genera. Los tamaños del efecto obtenidos para dichas comparaciones oscilan entre un tamaño del efecto medio ( $\mathrm{g}_{\text {Hedges }}=0,67$ para la comparación entre miedo a un atraco o robo y miedo al aumento de la llegada de inmigrantes) y un tamaño del efecto grande $\left(\mathrm{g}_{\text {Hedges }}=0,87\right.$ para la comparación entre miedo a sufrir un accidente de tráfico y miedo al aumento de la llegada de inmigrantes).

Esta escala tiene un índice de fiabilidad interesante, presentando un coeficiente alfa de $0,86 \mathrm{y}$, por otra parte, el análisis factorial proporciona un único factor (Tabla 4 en el apéndice). Así, se convierte en una escala muy práctica como posible indicador específico de Miedo al Delito. En este caso, cabe destacar que el ítem 3, miedo a sufrir un atraco o una agresión en la calle, es el que más peso tiene en el factor. Este indicador de Miedo al Delito presenta una correlación de $0,17(\mathrm{p}=0,000)$ con el factor Percepción de Peligro de la escala de Tipología de lugares peligrosos.

Atendiendo a la tipología sociodemográfica de los barrios también hemos podido encontrar diferencias estadísticamente significativas. El análisis de la varianza mostró un efecto principal estadísticamente significativo $(\mathrm{F}(2,1376)=3,5 ; \mathrm{p}=0,000)$ del tipo de barrio con relación al Miedo al Delito. La prueba de comparaciones múltiples HSD de Tukey reveló la existencia de diferencias estadísticamente significativas entre Barrio Social Alto (media= 27,7) en cuyos residentes se detecta mayor miedo al delito que en los de Barrio Social Bajo (media=29,3). El Barrio Social Medio (media= 28,4) no presenta diferencias significativas respecto a los anteriores en el miedo al delito. Nos encontramos, en fin, ante otra suerte de paradoja en la que se puede constatar que en los barrios de clase social alta, donde estadísticamente se dan unos índices de delincuencia más bajos, son, precisamente, donde la población manifiesta un mayor temor a ser víctima de un delito.

\section{4. ¿Por qué la gente tiene miedo?: hacia un modelo explicativo del miedo al delito.}

Uno de los objetivos de este estudio consiste, no solo en describir las escalas que presentamos de calidad de vida urbana y de miedo al delito, sino también nos gustaría intentar encontrar explicaciones a dicho fenómeno.

La cuestión consiste en determinar qué es lo que nos hace sentir este temor, más allá de los aspectos irracionales que la propia naturaleza del miedo conlleva. Para ello, realizaremos un repaso de las diferentes perspectivas teóricas desde las que se pretende explicar el miedo al delito. Antes de presentar los diferentes resultados obtenidos debe indicarse que todos los análisis planteados cumplieron los supuestos del modelo estadístico.

Desde el modelo de victimación el miedo al delito vendría explicado por el hecho de haber sido previamente víctima de un delito y por el impacto emocional que produjo

Revista Española de Investigación Criminólogica

REIC AC-01-05

http://www.criminologia.net

ISSN 1696-9219 
el mismo. Los resultados obtenidos (véase Tabla 5 en el apéndice) indican que, a pesar de que ambas variables resultaron estadísticamente significativas, este modelo no resulta adecuado para explicar el miedo al delito debido al escaso valor del tamaño del efecto obtenido, tal y como lo constata el coeficiente de determinación con un valor de 0,025 .

Por su parte, desde el modelo de control social y ambiental, el miedo al delito se explicaría en función del sentido de pertenencia, la actividad social o la satisfacción con el barrio. Como se muestra en la tabla 6 en el apéndice, sólo el sentido de pertenencia al barrio resultó estadísticamente significativo. Al igual que en el modelo previamente expuesto, el tamaño del efecto obtenido fue muy pequeño $\left(R^{2}=0,009\right)$ lo cual nos indica que tampoco este modelo resulta adecuado en nuestro caso para explicar el miedo al delito.

Finalmente, proponemos un modelo de acción razonada como modelo explicativo del miedo al delito. Desde esta teoría, el sujeto otorgaría explicaciones racionales al miedo al delito. Como puede observarse en la tabla 7 en el apéndice, este modelo es el que presenta un mejor ajuste, explicando un $31 \%$ de la varianza.

Al realizar un análisis de regresión, podemos comprobar las variables que quedan fuera de la ecuación (Tabla 4 en el apéndice) apareciendo como predictores: la preocupación general por los problemas sociales, la estimación de la probabilidad de que un familiar sea víctima de un delito preocupante, el grado de satisfacción con la Política de seguridad del Gobierno Central, el grado de eficacia de la Policía Autónoma y, finalmente, el Nivel de Instrucción y el género. Todos los valores del estadístico beta estandarizado son positivos, salvo obviamente, en el caso del grado de eficacia de la Policía Autónoma que cuanto mayor se considera dicha eficacia, menor es el miedo al delito, y, por otra parte, el Nivel de Instrucción, en el sentido de que a medida que aumenta la formación educativa, el miedo al delito disminuye.

\section{Conclusiones}

La literatura criminológica ha ofrecido diferentes aproximaciones para explicar el miedo al delito, que Gabriel \& Greve (2003) resumen en tres líneas fundamentales: $(a)$ Percepción de riesgo, (b) Vulnerabilidad y (c) Experiencia como víctima. Desde este punto de vista, la primera conclusión que podemos extraer es el cuestionamiento de los modelos teóricos de victimación, ya que el hecho de haber sido víctima de un delito previamente y su impacto emocional no presentaron tamaños del efecto adecuados en nuestro estudio. También pierden poder predictivo el modelo de control social y ambiental, ya que tampoco son decisivos en su aportación a la varianza explicada variables como el sentido de pertenencia, la actividad social o la satisfacción con el barrio.

También quedó demostrado anteriormente la debilidad del modelo de vulnerabilidad, ya que, por una parte, la supuesta mayor percepción de riesgo en las mujeres está justificada, en el caso de nuestra muestra, al conformar un colectivo que, según nuestros datos, resultó ser más victimizado que los varones $\mathrm{y}$, por otra parte, tampoco existe correlación entre edad y miedo al delito $(0,07)$. Así las cosas, las posibilidades de responder a la pregunta inicial se pueden acotar. Podría retomarse, en el caso que nos ocupa, lo que los psicólogos sociales conocemos como un modelo de acción razonada, que vendría a otorgar un perfil racionalista a las explicaciones del miedo al delito en la población entrevistada. En todo caso debemos ser prudentes a la

Revista Española de Investigación Criminólogica

REIC AC-01-05

http://www.criminologia.net

ISSN 1696-9219 
hora de descartar modelos explicativos del miedo al delito. Los anteriormente descritos han podido mostrarse poco reveladores en el contexto social y político objeto del estudio que nos ocupa, pero en otro escenario con diferentes políticas de seguridad, con mayor presencia de población itinerante o con otro perfil urbano, podemos encontrar explicaciones distintas. Esta relatividad limita de forma importante la validez externa de este estudio.

Nos encontramos, en fin, algo lejos de explicaciones relacionadas con procesos afectivos y más cerca de variables tan específicas como la estimación de la validez de políticas concretas de gestión de la seguridad. Esta parece ser la clave que debemos analizar para responder por qué la gente puede llegar a tener miedo o, al menos, es lo que parece desprenderse a la vista de los datos que nos ofrece este estudio realizado con población urbana.

En todo caso, en función de las necesidades de investigación, que pueden estar orientadas a la evaluación de escenarios urbanos o bien al análisis de la percepción de inseguridad asumido como indicador de calidad de vida urbana consideramos que pueden ser útiles las diferentes escalas que aquí se han presentado.

Desde este punto de vista, lo que estamos defendiendo, en fin, es un instrumento multifactorial desde perspectivas teóricas diversas. El diseño de una herramienta que tenga en cuenta todos los factores mencionados servirá para profundizar y explicar, no sólo la cuestionada paradoja del miedo al delito, sino lo que es más importante, los mecanismos psicosociales desde los cuales se construye lo que podríamos definir como la representación social del miedo. Este análisis va a ser el único realmente eficaz para diseñar una política de seguridad que devuelva los espacios públicos a los ciudadanos y ciudadanas, a los jóvenes y ancianos, a los niños y adultos, etc. y que, en definitiva, redunde en un aumento de la democratización del espacio, la calidad de vida urbana o, dicho de otra manera, la felicidad de las personas.

\section{BIBLIOGRAFIA}

Amérigo, M. (1995): Satisfacción residencial: Un análisis psicológico de la vivienda y su entorno. Madrid: Alianza Universidad.

Díez Ramos, M.A. (1996): "Seguridad ciudadana, victimización y opinión pública", Ciencia Policial, 37, Madrid, 25-43.

Ditton, J. \& Farral, S. (2000): The Fear of Crime. Dartmouth: Ashgate.

Eve, S.B. (1985): "Criminal victimization and fear of crime among the noninstitutionalized elderly in the United States: a critique of the empirical research literature", Victimology, 10, Arlington, 397-408.

Fattah, E. (1993): "Research on fear of crime: some common conceptual and measurement problems", Fear of crime and criminal victimization, (Bilski, Pfeiffer, Wetzels (comps.), Sttutgart, F. Enke, 282 pp.

Fattah, E.; Sacco, V.F. (1989): Crime and victimization of the elderly, New York, Springer-Verlag.

Fernández Ramírez, B. \& Corraliza, J.A. (1996): Aspectos físicos y sociales en los lugares peligrosos. Miedo al delito en un espacio institucional. Revista de Psicología Social, 11, 2, 219-234. 
Fernández Ramírez, B. \& Corraliza, J.A. (1997): Hacia una tipología de lugares peligrosos, en relación con el miedo al delito. Intervención Psicosocial, 6, 237-248.

Gabriel, U. \& Greve, W. (2003): The Psychology of Crime. British Journal of Criminology, 43, 600-614.

Hale, C. (1996): "Fear of crime: a review of the literature", International review of Victimology, 4, Bicester, 79-150.

Kury, H. \& Ferdinand, T. (1999): "Miedo al delito, tamaño de la población, salidas a la calle y actitudes hacia la Policía. Resultados alemanes", Revista de Derecho penal y Criminología, 3, Madrid, 209-292.

Lindquist, J.H.; Duke J.M. (1982): "The elderly victim at risk: explaining de fear victimization paradox", Criminology, 20, St. Louis, 115-126.

Keane, C. (1992): Victimization and Fear: Assessing the Role of Offender and Offence. Special Issue: Focus on the Violence Against Women Survey. Canadian Journal of Criminology, 37: 431-455.

Lofland, J. (1973): Analyzing social settings. New York: Belmont Books.

Naredo, M. (2000): Seguridad urbana y miedo al delito. Documentación Social, 119, 137-156.

San Juan, C. (2000): Theories of Design and Designs of Theory in Environmental Interventions. Bulletin of People-Environment Studies, $\mathrm{n}^{\mathrm{o}} 15$.

San Juan, C. \& Ocariz, E. (2001): El medio urbano como hábitat: patologías psicosociales y tratamientos ecourbanísticos. En J.L. GONZÁLEZ (Coord.): Sociedad y sucesos vitales extremos. Burgos: Servicio de publicaciones de la Universidad de Burgos.

Stafford, M.C. \& Galle, O.R. (1984): Victimization rates, exposure to risk and fear of crime. Criminology, 22, 173-185

Warr, M. (1984): "Fear of victimization: why are women and the elderly more afraid?", Social Science Quarterly, 65, Houston, 681-702.

César San Juan es doctor en Psicología social porla Universidad del País Vasco. Es subdirector de Investigación del Instituto Vasco de Criminología donde los autores desarrollan las siguientes líneas de investigación: Política criminal y prácticas penales, Delincuencia juvenil y de menores, Ecología del delito, Percepción de inseguridad, miedo al delito y prevención de la delincuencia, Ciencia policial y forense, y Victimología y justicia restaurativa.

iD https://orcid.org/0000-0001-8067-6729

Anabel Vergara es doctora en Psicología Social por la Universidad Católica de Lovaina (Bélgica).

iD https://orcid.org/0000-0002-3645-777X

Isabel Germán es Licenciada en Derecho y Master en Criminología en la Universidad del País Vasco.

iD https://orcid.org/0000-0002-2483-4187

Revista Española de Investigación Criminólogica 
Tabla 1. Análisis factorial de Satisfacción con el barrio

\begin{tabular}{|c|c|c|c|c|}
\hline$\overline{\text { Items }}$ & $F . I$ & F.II & F. III & $F . I V$ \\
\hline $\begin{array}{l}\text { Los vecinos en general } \\
\text {. }\end{array}$ & 88 & & & \\
\hline Con el barrio en general &, 82 & & & \\
\hline La vivienda en la que reside & ,79 & & & \\
\hline Centros escolares & &, 74 & & \\
\hline Transporte público & & ,67 & & \\
\hline Tiendas y mercados & & ,64 & & \\
\hline Parques y Zonas ajardinadas & & & ,83 & \\
\hline Zonas peatonales & & & ,79 & \\
\hline Lugares de ocio y recreo & & &, 53 & \\
\hline Servicio de limpieza en las calles & & & &, 79 \\
\hline Seguridad y vigilancia en las calles & & & &, 76 \\
\hline
\end{tabular}

Var. Total Explicada: 66\%

Revista Española de Investigación Criminólogica 
Tabla 2. Análisis factorial de Tipología de lugares peligrosos

\begin{tabular}{|c|c|c|c|}
\hline Items & F.I & F.II & F.III \\
\hline Es un lugar donde se suelen cometer delitos. &, 80 & & \\
\hline Normalmente suele haber gente con "mala pinta". &, 80 & & \\
\hline $\begin{array}{l}\text { Al pasar por ese lugar a veces pienso que puedo ser víctima } \\
\text { de un delito }\end{array}$ &, 69 & & \\
\hline En caso de peligro es probable que nadie me prestase ayuda &, 61 & & \\
\hline $\begin{array}{l}\text { Alguna parte del lugar puede ser un escondite para un } \\
\text { delincuente. }\end{array}$ &, 58 & & \\
\hline $\begin{array}{l}\text { Es un lugar muy deteriorado físicamente, muy maltratado, } \\
\text { abandonado. }\end{array}$ & & ,48 & \\
\hline $\begin{array}{l}\text { Es un lugar solitario, donde no hay actividad comercial, ni } \\
\text { anda gente. }\end{array}$ & &, 83 & \\
\hline No hay suficiente iluminación. & &, 81 & \\
\hline No existen medidas de seguridad., vigilancia, verjas, etc. & & ,67 & \\
\hline No hay caminos o formas de escapar en caso de peligro. & & ,61 & \\
\hline Es un lugar por el que debo pasar habitualmente. & & & ,91 \\
\hline
\end{tabular}

Var. Total Explicada: 62\% 
Tabla 3. Análisis de la varianza de medidas repetidas de los ítems de la escala de miedo al delito.

Estadísticos descriptivos

\begin{tabular}{|l|r|r|r|}
\hline & \multicolumn{1}{|c|}{ Media } & Desv. típ. & \multicolumn{1}{c|}{$\mathrm{N}$} \\
\hline Actos de violencia juvenil & 5,51 & 3,064 & 1451 \\
Acciones terroristas & 6,23 & 3,245 & 1451 \\
Atraco o agresión en la calle & 6,04 & 2,865 & 1451 \\
Robo en el domicilio & 6,04 & 2,964 & 1451 \\
Sufrir un accidente de tráfico & 6,46 & 2,715 & 1451 \\
Aumento de la llegada de inmigrantes & 4,56 & 3,036 & 1451 \\
Problema de salud por mala calidad de & 5,02 & 3,063 & 1451 \\
alimentos & & & \\
\hline
\end{tabular}

Pruebas de efectos intra-sujetos.

Medida: MEASURE 1

\begin{tabular}{|c|c|c|c|c|c|c|c|c|}
\hline Fuente & & $\begin{array}{c}\text { Suma de } \\
\text { cuadrados } \\
\text { tipo III }\end{array}$ & $\mathrm{gl}$ & $\begin{array}{c}\text { Media } \\
\text { cuadrática }\end{array}$ & $F$ & Significación & $\begin{array}{l}\text { Parámetro de } \\
\text { no centralidad }\end{array}$ & $\begin{array}{l}\text { Potencia } \\
\text { observada }^{a}\end{array}$ \\
\hline \multirow[t]{4}{*}{ factor1 } & Esfericidad asumida & 4182,616 & 6 & 697,103 & 145,156 & ,000 & 870,938 & 1,000 \\
\hline & Greenhouse-Geisser & 4182,616 & 5,180 & 807,390 & 145,156 &, 000 & 751,971 & 1,000 \\
\hline & Huynh-Feldt & 4182,616 & 5,201 & 804,164 & 145,156 & ,000 & 754,987 & 1,000 \\
\hline & Límite-inferior & 4182,616 & 1,000 & 4182,616 & 145,156 & ,000 & 145,156 & 1,000 \\
\hline \multirow[t]{4}{*}{ Error(factor1) } & Esfericidad asumida & 41781,098 & 8700 & 4,802 & & & & \\
\hline & Greenhouse-Geisser & 41781,098 & 7511,608 & 5,562 & & & & \\
\hline & Huynh-Feldt & 41781,098 & 7541,732 & 5,540 & & & & \\
\hline & Límite-inferior & 41781,098 & 1450,000 & 28,815 & & & & \\
\hline
\end{tabular}

a. Calculado con alfa $=, 05$ 
Tabla 4. Análisis factorial de Miedo a ser Víctima de un Delito.

\begin{tabular}{l|c}
\hline Items & F.I \\
\hline Los actos de violencia juvenil. &, 80 \\
Las acciones terroristas. &, 75 \\
Un atraco o agresión en la calle. &, 86 \\
Un robo en su domicilio. &, 83 \\
La posibilidad de sufrir un accidente de tráfico. &, 61 \\
El aumento de la llegada de inmigrantes. &, 61 \\
Un problema de salud por la mala calidad de los alimentos. &, 68 \\
\hline
\end{tabular}

Var. Total Explicada: 55\%

Tabla 5. Análisis de regresión para el modelo de victimación.

\begin{tabular}{|c|c|c|c|c|c|}
\hline \multicolumn{6}{|c|}{ Resumen del modeld } \\
\hline Modelo & $\mathrm{R}$ & R cuadrado & $\begin{array}{l}\text { R cuadrado } \\
\text { corregida }\end{array}$ & $\begin{array}{l}\text { Error típ. de la } \\
\text { estimación }\end{array}$ & $\begin{array}{l}\text { Durbin- } \\
\text { Watson }\end{array}$ \\
\hline 1 &, $161^{a}$ & 026 & 025 & 15,250 & 1,650 \\
\hline
\end{tabular}

\begin{tabular}{|c|c|c|c|c|c|c|c|c|}
\hline \multicolumn{9}{|c|}{ Coeficientes $^{\mathrm{a}}$} \\
\hline \multirow[b]{2}{*}{ Modelo } & & \multicolumn{2}{|c|}{$\begin{array}{l}\text { Coeficientes no } \\
\text { estandarizados }\end{array}$} & \multirow{2}{*}{ 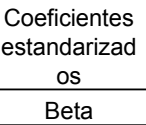 } & \multirow[b]{2}{*}{$\mathrm{t}$} & \multirow[b]{2}{*}{ Sig. } & \multicolumn{2}{|c|}{$\begin{array}{l}\text { Estadísticos de } \\
\text { colinealidad }\end{array}$} \\
\hline & & B & Error típ. & & & & Tolerancia & FIV \\
\hline 1 & (Constante) & 39,178 & ,443 & & 88,339 &, 000 & & \\
\hline & numero delitos sufridos & $-4,673$ & 1,483 &,- 229 & $-3,151$ & ,002 & 127 & 7,869 \\
\hline & impacto emocional & ,870 & 179 & ,353 & 4,858 & ,000 & 127 & 7,869 \\
\hline
\end{tabular}

a. Variable dependiente: miedo al delito 
Tabla 6. Análisis de regresión para el modelo de control social y ambiental.

\begin{tabular}{|l|r|r|r|r|r|}
\hline Resumen del modeld \\
\hline Modelo & $\mathrm{R}$ & $\mathrm{R}$ cuadrado & $\begin{array}{c}\text { R cuadrado } \\
\text { corregida }\end{array}$ & $\begin{array}{c}\text { Error típ. de la } \\
\text { estimación }\end{array}$ & $\begin{array}{c}\text { Durbin- } \\
\text { Watson }\end{array}$ \\
\hline 1 &, $107^{\mathrm{a}}$ &, 011 &, 009 & 15,292 & 1,862 \\
\hline
\end{tabular}
a. Variables predictoras: (Constante), Actividad social, Satisfacción general,
Sentido de pertenencia
b. Variable dependiente: Miedo al delito

\begin{tabular}{|c|c|c|c|c|c|c|c|c|}
\hline \multicolumn{9}{|c|}{ Coeficientes $^{a}$} \\
\hline & & \multicolumn{2}{|c|}{$\begin{array}{l}\text { Coeficientes no } \\
\text { estandarizados }\end{array}$} & $\begin{array}{c}\text { Coeficientes } \\
\text { estandarizad } \\
\text { os } \\
\end{array}$ & \multirow[b]{2}{*}{$\mathrm{t}$} & \multirow[b]{2}{*}{ Sig. } & \multicolumn{2}{|c|}{$\begin{array}{c}\text { Estadísticos de } \\
\text { colinealidad }\end{array}$} \\
\hline \multicolumn{2}{|c|}{ Modelo } & B & Error típ. & Beta & & & Tolerancia & FIV \\
\hline \multirow[t]{4}{*}{1} & (Constante) & 36,224 & 2,473 & & 14,650 & 000 & & \\
\hline & Satisfacción general &,- 031 & ,032 &,- 028 &,- 980 & ,328 & ,910 & 1,099 \\
\hline & Sentido de pertenencia & ,644 & , 190 & 099 & 3,383 & ,001 & ,899 & 1,112 \\
\hline & Actividad social & ,212 & , 195 & ,032 & 1,089 & ,276 & ,900 & 1,111 \\
\hline
\end{tabular}

a. Variable dependiente: Miedo al delito

Tabla 7. Análisis de regresión para el modelo de acción razonada.

\begin{tabular}{|l|r|r|r|r|c|}
\multicolumn{7}{|c|}{ Resumen del modeld } \\
\hline Modelo & $\mathrm{R}$ & $\mathrm{R}$ cuadrado & $\begin{array}{c}\text { R cuadrado } \\
\text { corregida }\end{array}$ & $\begin{array}{c}\text { Error típ. de la } \\
\text { estimación }\end{array}$ & $\begin{array}{c}\text { Durbin- } \\
\text { Watson }\end{array}$ \\
\hline 1 &, $557^{\text {a }}$ &, 311 &, 309 & 12,542 & 1,746 \\
\hline
\end{tabular}

Coeficientes $^{\mathrm{a}}$

\begin{tabular}{|c|c|c|c|c|c|c|c|c|}
\hline \multirow{2}{*}{\multicolumn{2}{|c|}{ Modelo }} & \multicolumn{2}{|c|}{$\begin{array}{l}\text { Coeficientes no } \\
\text { estandarizados }\end{array}$} & \multirow{2}{*}{$\begin{array}{c}\begin{array}{c}\text { Coeficientes } \\
\text { estandarizad } \\
\text { os }\end{array} \\
\text { Beta }\end{array}$} & \multirow[b]{2}{*}{$\mathrm{t}$} & \multirow[b]{2}{*}{ Sig. } & \multicolumn{2}{|c|}{$\begin{array}{l}\text { Estadísticos de } \\
\text { colinealidad }\end{array}$} \\
\hline & & $B$ & Error típ. & & & & Tolerancia & FIV \\
\hline \multirow[t]{10}{*}{1} & (Constante) & 7,698 & 3,034 & & 2,537 & 011 & & \\
\hline & Género & 2,489 & ,843 & 083 & 2,954 & ,003 & ,923 & 1,084 \\
\hline & $\begin{array}{l}\text { Preocupación general } \\
\text { por problemas sociales }\end{array}$ & ,283 & ,025 & ,316 & 11,205 & ,000 & ,908 & 1,101 \\
\hline & $\begin{array}{l}\text { Probabilidad estimada } \\
\text { de ser víctima de delito }\end{array}$ & 2,035 & ,173 & ,326 & 11,780 & ,000 & ,944 & 1,060 \\
\hline & $\begin{array}{l}\text { Satisfacción con política } \\
\text { seguridad Ayto. }\end{array}$ &,- 267 & ,311 &,- 041 &,- 859 & ,390 & ,322 & 3,108 \\
\hline & $\begin{array}{l}\text { Satisfacción con política } \\
\text { seguridad G.Autónomo }\end{array}$ & ,072 & ,283 & ,011 & ,254 & ,800 & ,369 & 2,708 \\
\hline & $\begin{array}{l}\text { Satisfacción con política } \\
\text { seguridad G.Central }\end{array}$ & ,660 & ,225 & , 105 & 2,929 & ,003 &, 559 & 1,790 \\
\hline & $\begin{array}{l}\text { Satisfacción con } \\
\text { actuación jueces }\end{array}$ &,- 196 & ,204 &,- 032 &,- 959 & ,338 & ,655 & 1,526 \\
\hline & $\begin{array}{l}\text { Grado eficacia policía } \\
\text { autonómica }\end{array}$ &,- 610 & ,185 &,- 096 & $-3,300$ & ,001 & ,849 & 1,178 \\
\hline & Nivel estudios & $-1,207$ & ,299 &,- 110 & $-4,041$ &, 000 & ,970 & 1,031 \\
\hline
\end{tabular}

a. Variable dependiente: Miedo al delito

Revista Española de Investigación Criminólogica

REIC AC-01-05 http://www.criminologia.net

ISSN 1696-9219

https://doi.org/10.46381/reic.v3i0.22 